\title{
Comparación de la Tomografía Axial Computarizada con el estudio anatomopatológico en el estadiaje ganglionar del cáncer de pulmón
}

\author{
M. López Mata ${ }^{1}$, J. Valencia Julve ${ }^{1}$, N. Bascón Santalón ${ }^{1}$, C. Velilla Millán ${ }^{1}$, R. Escó Barón ${ }^{1}$, M. García Álvarez $^{2}$
}

\section{Resumen}

- Propósito: Evaluar la Sensibilidad, Especificidad, Valor predictivo positivo y Valor Predictivo Negativo de la Tomografia Axial Computarizada (TAC) en el estadiaje ganglionar del cáncer de pulmón.

- Material y métodos: Se han analizado 30 pacientes diagnosticados de carcinoma pulmonar entre Mayo de 2003 y Mayo de 2004. A todos los pacientes se les realizó una TAC torácica, valorando la afectación ganglionar mediastínica. A su vez, se les realizó o una mediastinoscopia o una resección pulmonar más linfadenectomía mediastínica, obteniendo así material para el estudio anatomo-patológico para confirmar o no la afectación ganglionar mediastínica y correlacionarla con los hallazgos de la TAC.

- Resultados: Hemos obtenido una Sensibilidad del 72,2\%, una Especificidad del 100\%, un valor predictivo positivo del $100 \%$ y un valor predictivo negativo del 70,6\% para la TAC, utilizando como "gold standar" el estudio anatomopatológico.

- Conclusiones: La TAC torácica se considera una prueba de imagen de rutina en el diagnóstico del cáncer de pulmón; pero en muchos casos no es capaz de estadiar correctamente la afectación ganglionar mediastínica. Es en estos casos, donde es necesario realizar pruebas invasivas como la mediastinoscopia. Actualmente, la aparición de la PET permite estadiar mejor el tumor, ofreciendo mejor tratamiento a cada paciente, y en determinados casos evitar técnicas diagnósticas invasivas.

\section{Palabras clave:}

Carcinoma pulmonar. TAC. Mediastinoscopia.

Oncología, 2005; 28 (7):343-350

\footnotetext{
${ }^{1}$ Servicio de Oncología Radioterápica. Hospital Clínico Universitario Lozano Blesa

${ }^{2}$ Servicio de Cardiología. Hospital Universitario Miguel Servet

Zaragoza
} 


\section{Summary}

- Purpose: To analise the sensitivity, specificity, and positive and negative predictive values of the computerized axial tomography (CT) in nodal stages of lung carcinoma.

- Material and methods: Thirty patients suffering from lung carcinoma were analysed between May 2003 and May 2004. CT screening of the thorax was made to all the patients. Mediastinoscopy or lung resection samples plus systematic node dissection were performed, and the efficiency of CT analysed by comparing the obtained data with the histopathology results of nodal affection shown by mediastinoscopy and lymph node dissection.

- Results: The sensitivity, specificity, and positive and negative predictive value of the computerized axial tomography (CT) for nodal staging involved in lung carcinoma were $72.2 \%, 100 \%, 100 \%$ and $70,6 \%$ respectively.

- Conclusions: CT of the thorax is considered a usual imaging technique in lung carcinoma diagnosis; but in some cases it does not focalise correctly the nodal staging involved in lung carcinoma. In theses cases, it is necessarry to perform invasive techniques such as mediastinoscopy. At present, positron emission tomography (PET) is the technique that better permits to focalise the tumor offering the best data for the therapy of each patient, and avoiding invasive diagnosis techniques.

Key words: Lung carcinoma. CT. Mediastinoscopy.

\section{Introducción}

El cáncer de pulmón es el tumor con mayor mortalidad en el mundo occidental. A pesar de los avances diagnósticos (TAC, PET, mediastinoscopia, etc) y terapéuticos (nuevos fármacos quimioterápicos, avances quirúrgicos y nueva tecnología en radioterapia), el cáncer de pulmón sigue presentando una elevada mortalidad $(<15 \%$ de los pacientes sobreviven a los 5 años tras el diagnóstico $)^{1}$.

Supone unos 18.500 casos nuevos al año en España, y en el año 2000, fue el responsable de 17308 muertes (CNE 2000). La razón hombre/mujer es de 4,5 en Europa y de 11 en España.

Existen muchos tipos histológicos de cáncer pulmonar. A nivel clínico, se reconocen básicamente dos tipos fundamentales: el carcinoma de células pequeñas o carcinoma microcítico y el carcinoma de células no pequeñas.

El pronóstico del cáncer de pulmón está íntimamente ligado a la afectación ganglionar presente, la cual marca la actitud terapéutica a seguir.

El estudio de extensión locorregional del cáncer de pulmón se ha basado clásicamente en la Tomografia Axial Computarizada.

La TAC nos permite:
- Determinar el grado de extensión de la neoplasia, valorando las adenopatías mediastínicas.

- Aportar información sobre la morfología del tumor, la presencia de cavitación, la localización del tumor y sus relaciones.

- Analizar las lesiones subpleurales y existencia de derrame pleural y/o pericárdico.

- Valorar la afectación de la pared costal.

La presencia de adenopatías mediastínicas con un diámetro corto mayor a $1 \mathrm{~cm}$ es altamente sospechosa de enfermedad secundaria por la mayoría de autores. Sin embargo, no hay acuerdo generalizado y en la literatura se describen criterios distintos de compromiso ganglionar como son: diámetro de eje mayor superior a $1 \mathrm{~cm}, 1,5 \mathrm{~cm}$ o $2 \mathrm{~cm}$, la existencia evidente de necrosis o la morfología de los bordes.

Existe un 5-15\% de lesiones histológicamente positivas para malignidad que en la TAC se presentan como adenopatías de menor de $0,5 \mathrm{~cm}$ de diámetro ${ }^{2-4}$ por lo que se consideran negativas al informar la TAC. De igual forma, hasta un $40 \%$ de los ganglios de mayor de $1 \mathrm{~cm}$. pueden ser histológicamente benignos (enfermedades infecciosas o reacción inflamatoria). 
La PET (Tomografia por Emisión de Positrones) es una técnica de diagnóstico que se basa en la actividad biológica de las células neoplásicas. Parece ser que la PET mejora la sensibilidad y especificidad de la TAC en el estudio de las adenopatías mediastínicas ${ }^{5-7}$. En la actualidad, se está generalizando el uso de la PET para el estadiaje del carcinoma de pulmón; aunque aun no se considera una técnica de rutina en nuestro medio.

Sin embargo, aún con PET positivo no se debe obviar el estudio histológico mediante mediastinoscopia previa a la cirugía ya que existen falsos positivos (por procesos inflamatorios) que, si no se realiza mediastinoscopia, quedarían excluidos de la cirugía, aun siendo pacientes potencialmente quirúrgicos. De igual forma, existen casos que no observándose afectación ganglionar por técnicas de imagen, sí se detecta en el estudio anatomopatológico 9 .

La mediastinoscopia, a pesar de ser una técnica invasiva, es la técnica más precisa (presenta una sensibilidad superior al $90 \%$ y una especificidad cercana al $100 \%)^{9,-12}$ para establecer el estadio tumoral de los ganglios del mediastino superior (N2 o N3), previa a la valoración terapéutica de los pacientes con cáncer de pulmón.

El objetivo de este estudio es evaluar la sensibilidad, especificidad, valor predictivo positivo y valor predictivo negativo de la TAC en el estadiaje ganglionar del cáncer de pulmón en nuestro medio, usando como "gold standar" el estudio anatomopatológico.

\section{Material y métodos}

Se han estudiado retrospectivamente 30 pacientes a los que se les realizó mediastinoscopia o resección pulmonar más linfadenectomía mediastínica, tras ser diagnosticados de cáncer de pulmón sin metástasis a distancia, entre el 1 de Mayo del 2003 y el 1 de Mayo del 2004 en el Hospital Clínico Universitario "Lozano Blesa" de Zaragoza.

A todos los pacientes ante la sospecha de cáncer de pulmón, se les realizó una TAC torácica; valorando la lesión pulmonar primaria y la afectación ganglionar mediastínica. Se consideraron patológicas o sospechosas de malignidad, aquellas adenopatías que presentaban un diámetro corto superior a $1 \mathrm{~cm}$.

Según el estadio clínico-radiológico, se realizó mediastinoscopia o resección tumoral con linfadenectomía mediastínica. En principio, en aquellos casos que la TAC identificaba adenopatías sospechosas de malignidad se realizaba mediastinoscopia. En los casos que la TAC no informaba de adenopatías sospechosas, el tratamiento de elección era la cirugía (resección pulmonar más linfadenectomía mediastínica), si la lesión primaria pulmonar lo permitía.

El estadiaje del cáncer de pulmón se ha realizado según la clasificación TNM (Adaptada de Mountain CF, Chest 1997; 111; 1710-23) ${ }^{13,14}$. La T hace referencia al tumor primario, la $\mathrm{N}$ a la afectación ganglionar y la M a la diseminación metastásica.

La afectación ganglionar incluye los ganglios linfáticos regionales (distribuidos por niveles ganglionares según Tabla I), que en el caso del cáncer de pulmón se distribuye de la siguiente forma:

N0 No metástasis en ganglios linfáticos regionales.

N1 Metástasis en ganglios linfáticos peribronquiales y/o hiliares homolaterales; ganglios intrapulmonares afectados por extensión directa del tumor.

N2 Metástasis en ganglios mediastínicos homolaterales y/o subcarinales.

N3 Metástasis en ganglios mediastínicos contralaterales, hiliares contralaterales, escalenos homo o contralaterales, o supraclaviculares.

TABLA I

\section{Niveles ganglionares mediastínicos}

\begin{tabular}{|cll|}
\hline Nivel & Localización & $\begin{array}{c}\text { Estadio } \\
\text { ganglionar }\end{array}$ \\
\hline 1 & Ganglios mediastínicos altos & \\
2 & Ganglios paratraqueales altos & \\
3 & Ganglios prevasculares y retrotraqueales & \\
4 & Ganglios paratraqueales bajos & $\mathrm{N} 2$ \\
5 & Ganglios subaórticos & \\
6 & Ganglios para-aórticos & \\
7 & Ganglios subcarínicos & \\
8 & Ganglios paraesofágicos & \\
9 & Ganglios del ligamento pulmonar & $\mathrm{N} 1$ \\
\hline 10 & Ganglios hiliares & \\
11 & Ganglios interlobares & \\
12 & Ganglios lobares & \\
13 & Ganglios segmentarios & \\
14 & Ganglios subsegmentarios & \\
\end{tabular}


La muestra ganglionar obtenida, ya fuera por mediastinoscopia como por linfadenectomía mediastínica, fue analizada histológicamente; obteniendo así el estadiaje histológico de todos los pacientes. No hemos tenido en cuenta si la realización de mediastinoscopia o linfadenectomía mediastínica era la actitud más indicada. Lo que nos interesaba era la obtención de material para el estudio anatomopatológico, y así obtener la correlación imagen-histología.

Se han calculado la sensibilidad, especificidad, valor predictivo positivo y valor predictivo negativo de la tomografía axial computarizada para el diagnóstico y estadiaje ganglionar mediastínico en el cáncer de pulmón; utilizando las siguientes fórmulas:

\section{Sensibilidad (S): VP/VP+FN \\ Especificidad (E): VN/VN+FP \\ Valor predictivo positivo (VPP): VP/VP+FP \\ Valor predictivo negativo (VPN): $\mathrm{VN} / \mathrm{VN}+\mathrm{FN}$}

Se ha considerado como método de referencia del estudio o "gold standar", el análisis anatomo-patológico de la muestra ganglionar; considerando:

Verdaderos positivos (VP): Aquellos pacientes que en la TAC se describían adenopatías sospechosas de malignidad que han sido confirmadas en el estudio anatomopatológico.

Verdaderos negativos (VN): Aquellos pacientes que en la TAC no se describían adenopatías mediastínicas patológicas y en el estudio histológico no se halló material neoplásico.

Falsos positivos (FP): Aquellos pacientes que en la TAC se describían adenopatías mediastínicas patológicas y en el material estudiado no se observan signos de malignidad.

Falsos negativos (FN): Aquellos pacientes que en la TAC no se describen adenopatías mediastínicas sospechosas de malignidad y en el estudio anatomopatológico se ha hallado material neoplásico.

\section{Resultados}

De los 30 pacientes incluidos en el estudio, en 10 casos se practicó la mediastinoscopia, en un caso se realizó una toracotomía exploradora y diagnóstica con el fin de obtener material para estudio histológico; y en los 19 casos restantes se practicó una resección pulmonar (lobectomía o neumonectomía) más linfadenectomía mediastínica.
TABLA II

T y N según TAC

\begin{tabular}{|cc|}
\hline$T$ & $N$ \\
\hline $\mathrm{T} 1=26,66 \%(8)$ & $\mathrm{N} 0=46,66 \%(14)$ \\
$\mathrm{T} 2=60 \%(18)$ & $\mathrm{N} 1=10 \%(3)$ \\
$\mathrm{T} 3=3,33 \%(1)$ & $\mathrm{N} 2=43,33 \%(13)$ \\
$\mathrm{T} 4=10 \%(3)$ & $\mathrm{N} 3=0$ \\
\hline
\end{tabular}

TABLA III

T y N según estudio anatomopatológico

\begin{tabular}{|cc|}
\hline$T$ & $N$ \\
\hline $\mathrm{T} 1=26,66 \%(8)$ & $\mathrm{N} 0=36,66 \%(11)$ \\
$\mathrm{T} 2=50 \%(15)$ & $\mathrm{N} 1=13,33 \%(4)$ \\
$\mathrm{T} 3=16,66 \%(5)$ & $\mathrm{N} 2=43,33 \%(13)$ \\
$\mathrm{T} 4=6,66 \%(2)$ & $\mathrm{N} 3=6,66 \%(2)$ \\
\hline
\end{tabular}

El diagnóstico anatomopatológico fue de un $50 \%$ (15) de los casos carcinoma escamoso, un $43,3 \%$ (13) adenocarcinoma, un 3,3\% (1) carcinoma de célula pequeña y otro $3,3 \%$ (1) tumor neuroendocrino.

La distribución de los estadios varía según se haga por TAC o por descripción anatomopatológica. Los resultados obtenidos en esta muestra se recogen en la Tabla II y Tabla III.

El $53,33 \%$ de los pacientes $(n=16)$ presentaron adenopatías torácicas sospechosas de malignidad según la TAC. En 3 casos, el estadiaje ganglionar según TAC correspondió a N1 y en 13 casos, se estadiaron como N2

La localización torácica más frecuente donde se describen adenopatías patológicas fue: nivel paratraqueal bajo homolateral $(56,25 \%)$, adenopatías hiliares homolaterales $(56,25 \%)$, adenopatías subcarínicas $(43,75 \%)$, adenopatías interlobares $(25 \%)$, nivel paratraqueal alto $(18,75 \%)$ y nivel prevascular $(12,5 \%)$.

De los 16 casos que había afectación ganglionar, según TAC, a 8 pacientes se les realizó mediastinoscopia, a 7 pacientes se le practicó resección pulmonar más linfadenectomía mediastínica y en un caso se realizó una toracotomía exploradora.

De los 10 casos en que se realizó mediastinoscopia, 6 casos presentaron afectación de los ganglios paratraqueales bajos homolaterales, en 3 casos se 
halló afectación de los ganglios subcarínicos, en un caso había afectación de los ganglios interlobares homolaterales y en uno de los casos había invasión de los ganglios paratraqueales bajos contralaterales.

De los 19 casos en que se practicó una resección pulmonar más linfadenectomía mediastínica, 9 presentaron afectación ganglionar al estudiar histológicamente la muestra. En estos casos, la localización ganglionar más frecuente fue el nivel paratraqueal bajo homolateral (en tres casos) y los ganglios interlobares homolaterales (en 4 casos).

En nuestra muestra, de los 30 pacientes, hemos obtenido 13 Verdaderos positivos; es decir casos en que la sospecha de adenopatías torácicas patológicas se ha confirmado con el estudio anatomo-patológico de la muestra quirúrgica. Ha habido 12 casos de verdaderos negativos (la TAC no mostraba adenopatías con diámetro corto mayor a $1 \mathrm{~cm}$ y en el estudio AP no se han observado signos de malignidad). Finalmente, hemos obtenido 5 casos donde la TAC no describía afectación ganglionar sospechosa pero el estudio anatomopatológico ha resultado positivo para malignidad; lo que representa un $16,6 \%$ de falsos negativos. No hemos obtenido ningún caso de falso positivo.

Con estos datos y siguiendo las fórmulas que se describen previamente, hemos obtenido la sensibilidad, especificidad, valor predictivo positivo y negativo para la TAC.

\section{Sensibilidad $=72$ '2\% \\ Especificidad $=100 \%$ \\ $\mathrm{VPP}=100 \%$ \\ $\mathrm{VPN}=70$ '6\%}

Dada la importancia de la estación ganglionar afecta, de cara a la actitud terapéutica a seguir, hemos analizado la sensibilidad y especificidad para los niveles ganglionares más frecuentemente afectos, que se resume en la Tabla IV.

TABLA IV

Sensibilidad y especificidad según nivel ganglionar

\begin{tabular}{|lcc|}
\hline Nivel ganglionar & Sensibilidad & Especificidad \\
\hline Nivel 10 y 11 & $60 \%$ & $79 \%$ \\
Nivel 4 & $80 \%$ & $95 \%$ \\
Nivel 7 & $60 \%$ & $84 \%$ \\
\hline
\end{tabular}

Si analizamos los grupos según la técnica quirúrgica utilizada, obtenemos que:

Se han realizado 19 resecciones pulmonares con linfadenectomía mediastínica. De los 19 casos, en la TAC se detectaron adenopatías sospechosas de malignidad en 4 pacientes las cuales se confirmaron histológicamente. Hubo otros 4 casos, en que la TAC no describía adenopatías mayores de $1 \mathrm{~cm}$, y al realizar la disección ganglionar se observó afectación tumoral. Por lo tanto en nuestro estudio, 4 de 19 casos pasaron de estadio $\mathrm{N}-\mathrm{a}+\mathrm{N}+$ al realizar el estudio histológico. Esto representa un $21 \%$ de los casos en que la TAC no ha revelado adenopatías sospechosas de malignidad, que se confirmaron como malignas en el estudio AP.

En los casos de mediastinoscopia, se realizaron 10 en total, hubo un caso que la TAC no detectó afectación ganglionar que se confirmó como $\mathrm{N}+$ en el estudio AP. En el resto de las 9 exploraciones mediastínicas, la TAC y el estudio histológico coincidieron (8 casos positivos para malignidad en anatomía patológica como en la TAC, y un caso con histología negativa y TAC no sospechosa de afectación ganglionar).

En el caso que se realizó toracotomía, la TAC torácica informaba de adenopatía sospechosa a nivel 4R; que se confirmó con el estudio AP.

\section{Discusión}

El cáncer de pulmón es uno de los tumores malignos con mayor mortalidad en el mundo y representa la primera causa de muerte por cáncer en el sexo masculino.

Por el momento, los esfuerzos que se han realizado para conseguir desarrollar un programa de detección precoz no han dado buenos resultados ${ }^{11,15-18}$.

Tras la detección de una masa pulmonar debemos realizar un estudio exhaustivo del mediastino,; ya que la presencia o no de afectación ganglionar marcará la actitud terapéutica a seguir y por lo tanto el pronóstico. En estadios iniciales (I, II) y algunos estadios locamente avanzados (IIIA excepto N2) del cáncer de pulmón del tipo no-célula pequeña está indicada la cirugía. Cuando no puede usarse la cirugía como arma terapéutica, ya sea por el estadio de la enfermedad (T4, invasión pleural, afectación ganglionar N2) como por el estado general del paciente, la supervivencia se reduce notablemente. 
Ante la sospecha clínica y/o por radiografía simple de tórax de carcinoma pulmonar, se debe realizar una TAC torácica para determinar el tamaño y la extensión del tumor y valorar la afectación mediastínica. El examen mediante TAC suele aportar información bastante exacta del tamaño y la extensión local del tumor, pero la afectación ganglionar suele ser más difícil de valorar.

Existen múltiples estudios ${ }^{10,19-22}$ que han intentado determinar la sensibilidad y especificidad de la TAC para el estadiaje ganglionar del cáncer de pulmón, obteniendo resultados no siempre congruentes. Una de las razones por la que existen estas diferencias es la decisión del tamaño que debe tener una adenopatía para considerarla sospechosa de malignidad.

En nuestro estudio, hemos considerado adenopatía sospechosa de malignidad mediante valoración por TAC; aquella que presentaba un tamaño superior o igual a $10 \mathrm{~mm}$ de diámetro corto. Considerando este tamaño, hemos obtenido una sensibilidad del $72,2 \%$ para la TAC en el estadiaje del cáncer de pulmón. Esta cifra es similar a la obtenida por otros autores $(61-77 \%)^{5,10,21-23}$. Existen autores ${ }^{22}$ que consideran que una adenopatía es sospechosa de malignidad cuando mide más ó igual a $15 \mathrm{~mm}$. En estos casos, la sensibilidad que se obtiene es mayor (79$82 \%)$.

La especificidad de la TAC en el estadiaje ganglionar del cáncer de pulmón en nuestro estudio ha sido del 100\%; es decir la probabilidad de clasificar correctamente un individuo sin afectación ganglionar es del $100 \%$. En la literatura consultada, este valor es más variable que la sensibilidad, oscilando entre $57 \%$ y el $100 \%$ según estudios ${ }^{10,19,21}$.

A nivel de práctica clínica, más que la sensibilidad y la especificidad, son útiles el valor predictivo positivo y el negativo. Estos dos conceptos nos dan más seguridad de la prueba estudiada. En nuestro estudio hemos obtenido un VPP del 100\% (en la literatura $^{10,21}$ oscila entre $85-100 \%$ ) y un VPN del $70,6 \%$, es decir; existe una probabilidad del $100 \%$ de que un paciente presente afectación ganglionar, si se obtiene un resultado positivo en la TAC y existe una probabilidad del $70,6 \%$ de que un paciente no presente afectación ganglionar, cuando el resultado es negativo.

Según el nivel ganglionar mediastínico afecto, hemos encontrado pequeñas variaciones en cuanto la sensibilidad y especificidad de la TAC para detectar afectación ganglionar. En nuestro estudio, el nivel que más frecuentemente ha estado afecto ha sido el nivel 4 y los niveles 10 y 11, con una frecuencia del $56,25 \%$. La sensibilidad y especificidad de la TAC para detectar adenopatías en nivel 4 han sido del $80 \%$ y $95 \%$ respectivamente; valores algo superiores a los encontrados en la literatura ${ }^{23}(\mathrm{~S}=60 \%$ y $\mathrm{E}=86 \%$ ). La sensibilidad de la TAC para detectar afectación ganglionar subcarinal ha sido del $60 \%$ y la Especificidad del 84\%. En otros estudios ${ }^{23}$, 24, la sensibilidad y especificidad oscilan entre $25-47 \%$ y $83-91 \%$, respectivamente. Tanto nuestro estudio, como el de estos otros autores demuestran la baja sensibilidad de la TAC para la identificación de afectación ganglionar subcarinal.

Tanto los datos obtenidos en nuestro estudio como los obtenidos por otros autores, nos plantean la necesidad de seguir realizando exploraciones mediastínicas invasivas.

Existen autores ${ }^{11}$ que defienden el uso de la exploración mediastínica del carcinoma pulmonar de forma sistemática; independientemente de los hallazgos de la TAC. El principal argumento de esta opinión es que hasta el 17\% de los casos que la TAC no ha revelado adenopatías mayores de $1 \mathrm{~cm}$ presentan afectación ganglionar al estudio anatomo-patológico de la pieza quirúrgica. En nuestro estudio, hemos obtenido un 16,6\% de falsos negativos (pacientes que no presentan adenopatías en el estudio por TAC y que el estudio anatomo-patológico demuestra afectación ganglionar), valor muy similar al hallado en la literatura.

Actualmente, la aparición del PET permite detectar o confirmar la sospecha de adenopatías mediastínicas que la TAC no es capaz de identificar. Según estudios $^{6,7,10,22,25}$ realizados, esta prueba presenta mayor sensibilidad (85-94\%) y especificidad (80$90 \%$ ) que la TAC. Y de igual forma que en la TAC, si el tamaño considerado sospechoso de malignidad es mayor de $15 \mathrm{~mm}$, la sensibilidad del PET aumenta. Aunque aun no se considera un prueba de imagen de rutina en el diagnóstico del cáncer de pulmón; en casos donde la TAC presenta dudas de afectación ganglionar, el PET podría ayudarnos a estadiar correctamente el tumor y así poder ofrecer el mejor tratamiento para cada paciente, evitando las técnicas diagnósticas invasivas.

Existen numerosos estudios que han analizado la 
sensibilidad, especificad, VPP y VPN de la TAC en el estadiaje ganglionar del cáncer de pulmón; usando como método de referencia el estudio anatomopatológico de la muestra ganglionar. Con nuestro estudio, hemos pretendido analizar nuestros propios resultados. A pesar de que es un estudio con una muestra pequeña (30 pacientes) y se ha comparado la TAC con el estudio anatomopatológico tanto de muestra obtenida por mediastinoscopia como de la muestra obtenida por resección pulmonar y linfadenectomía mediastínica, nuestros resultados són similares a los obtenidos por otros autores.

\section{Correspondencia:}

Dra. M. López Mata

Servicio de Oncología Radioterápica

Hospital Clínico Universitario Lozano Blesa

C/ San Juan Bosco, 15

E-50009 Zaragoza

E-mail: mlopezm76@hotmail.com

\section{Bibliografía}

1. Lopez-Abente G, Pollán M, et al. Informe sobre la salud de los españoles. Cáncer. Información disponible a Diciembre de 2003.

2. Orestes N, Mederos Curbelo, Chirino CP, et al. Variación del estadiamiento clínico y el posquirúrgico en el cáncer pulmonar no células pequeñas. Rev Cubana Oncol 2000; 16 (3): 146-9.

3. Ginsberg RJ, Rubinstein LV. Randomizedtrial of lobectomy versus limited resection for T1 N0 non-small lung cancer. Lung Cancer Study Group. Ann Thorac Surg. 1995 Sep;60(3):615-22; discussion 622-3.

4. Gdeedo A, Van Schil P, Corthouts B, Van Mieghem, Van Meerbeeck J, Van Marck E. Comparison of imaging TNM [(i)TNM] and pathological TNM [(p)TNM] in staging of bronchogenic carcinoma. Eur J Cardiothorac Surg. 1997 Aug;12(2):224-7.

5. Halter G, Buck AK et al. Lymph node staging in lung cancer using [18F] FDG-PET. Thorac Cardiovascular Surgery 2004 April 52 (2); 96-101.

6. Graeter TP, Hellwig D et al. Mediastinal lymph node staging in suspected lung cancer: comparison of positron emission tomography with F-18-fluorodeoxyglucose and mediastinoscopy. Ann Thoracic Surgery 2003 Jan; 75 (1): 231-5; discusion 235-6.
7. Gonzalez-Stawunski GV, Lemaire A, Merchant F et al. A comparative analysis of positron emision tomography and mediastinoscopy in staging non-small cell lung cancer. J Thoracic Cardiovascular Surgery 2003 Dec; 126 (6): 17003.

8. Agencia de Evaluación de Tecnologías Sanitarias (AETS). Instituto de Salud Carlos III- Ministerio de Sanidad y Consumo. "Tomografía por Emisión de Positrones (PET) con 18-FDG en Oncología Clínica (Revisión Sistemática)". Madrid:AETS - Instituto de Salud Carlos III, Noviembre 2001.

9. Rami Porta R, Mateu Navarro M, González Pont G, Cuesta Palomero M. Resultados del tratamiento quirúrgico del carcinoma broncogénico N2 patológico con mediastinoscopia negativa. Archivos de Bronconeumología 2000 Julio; número 7- Volumen 36 p. 365-370.

10. Rodríguez P, Santana N, Gámez P, et al. La mediastinoscopia para el diagnóstico de la enfermedad: análisis de 181 exploraciones. Arch Bronconeumologia 2003; 39 (1): 2934.

11. Martin de Nicolás Serrahima J, García Barajas S, Marrón Fernández $\mathrm{C}$, et al. Complicaciones técnicas de la exploración quirúrgica del mediastino en la estatificación del cáncer de pulmón. Archivos de Bronconeumología 1999; 35:390-4.

12. Van Schil PE, Van Hee RH, Schoofs EL. The value of mediastinoscopy inpreoperative staging of bronchogenic carcinoma. J Thorac Cardiovasc Surg. 1989 Feb;97(2):240244.

13. Mountain CF: Revisions in the International System for Staging Lung Cancer. Chest 1997; 111: 1710-17.

14. Lung In: American Joint Committee on Cancer: AJCC Cancer Staging Manual. 6th ed. New York, NY: Springer, 2002, pp167-181.

15. Spiro SG. Lung tumours. En: Brewis RAL, Gibson GJ, Geddes DM (eds.). Textbook of Respiratory Medicine, Londres: Baillière Tindall, 1990; 832-879.

16. Carr DT, Holoye PY, Hong WK. Bronchogenic carcinoma. En: Murray JF, Nadel JA (eds.). Textbook of Respiratory Medicine, Filadelfia: Saunders, 1994; 1528-1596.

17. Garcés JM, Guasch Y, Planas J, Broquetas J, Drobnic L. Cáncer de pulmón. Análisis de 122 casos. Med Clin (Barc) 1982; 79: 441-444.

18. Michael A. Beckles, MB, BS; Stephen G. Spiro, MD; Gene L. Colice, MD, FCCP and Robin M. Rudd, MD. Initial Evaluation of the Patient with Lung Cancer. Chest. 2003; 123:97S-104S.

19. Thanos Sioris, Ritva Järvenpää et al. Comparison of computed and systematic lymph node dissection in determining TNM and stage in non-small cell lung cancer. European Journal od Cardio-thoracic Surgery 23 (2003) 403-408.

20. Erdogan Cetinkaya, Akif Turna et al. Comparison of clinical and surgical-pathologic staging of the patients with non-small cell lung carcinoma. European Journal od Cardio-thoracic Surgery 22 (2002) 1000-1005.

21. Staples CA, Muller NL, Millar RR et al. Mediastinal nodes 
M. López Mata y cols.

in bronchogenic carcinoma: comparison betweeen CT and mediastinoscopy. Radioology. 1988 May; 167(2): 367-72.

22. Breyer RH, Karstaedt N, Mills SA et al. Computed tomography for evaluation of mediastinal lymph nodes in lung cancer: correlation with surgical staging. Ann Thoracic Surgery 1984 Sep; 38 (3): 215-20.

23. Gdeedo A, Van Schil P, Corthouts B, Van Meerbeeck J, Van Marck E. Prospective evaluation of computed tomography and mediastinoscopy in mediastinal lymph node staging. Eur Respir J 1997; 10: 1547-1551.
24. McLoud TC, Bourgouin PM, Greenberg RW, et al. Bronchogenic carcinoma: analysis of staging in the mediastinum with CT by correlative lymph node mapping and sampling. Radiology 1992; 182: 319-323.

25. GouldMK, Kuschner WG, Rydzak CE, Maclean CC, Demas AN, et al. Test performance of positron emission tomography and computed tomography for mediastinal staging in patients with non-small-cell cancer: a meta-analysis. Ann Intern Med. 2003 Dec 2; 139(11):879-92. 